\title{
Factores asociados al consumo frecuente de marihuana en jóvenes antes de su ingreso a centros juveniles de diagnóstico y rehabilitación en Perú
}

\section{Factors associated with frequent marijuana consumption in young people before admission to juvenile detention centers in Peru}

\author{
Ali Al-Kassab-Córdova*, Gonzalo Cornejo-Venegas**, Nataly Gacharna-Madrigal**, \\ Carlos Baquedano-Rojas**, Guillermo De la Borda-Prazak**, Christian R. Mejia**. \\ * Centro de Excelencia en Estudios Económicos y Sociales en Salud, Universidad San Ignacio de Loyola, Lima, Perú. \\ ** Escuela de Medicina. Universidad Peruana de Ciencias Aplicadas, Lima, Perú.
}

\section{Resumen}

La marihuana es la droga ilícita más consumida en el mundo, especialmente entre jóvenes. El presente estudio es relevante para la toma de decisiones en salud porque expande el conocimiento sobre el uso de drogas en la juventud vulnerable y permite a las autoridades sanitarias reducir el consumo de marihuana mediante estrategias educativas, familiares y gubernamentales. El objetivo de este estudio fue determinar la prevalencia del consumo frecuente de marihuana y sus factores asociados en jóvenes antes de su ingreso a centros juveniles de diagnóstico y rehabilitación en Perú. Los datos fueron tomados del Censo Nacional de Población en los Centros Juveniles de Diagnóstico y Rehabilitación del año 2016 en Perú. La muestra final estuvo compuesta por 1848 personas entre 14 y 22 años, con una mediana de edad de 17 años (95,6 \% hombres). La variable consumo frecuente de marihuana fue definida como el consumo de marihuana de al menos una vez por semana por parte de los jóvenes, previo a su ingreso al centro. Los principales factores asociados al consumo frecuente de marihuana fueron el sexo masculino, huir de casa antes de los 15 años, haber sufrido abuso físico durante la infancia, tener un miembro de la familia que consuma alcohol o drogas frecuentemente y la presencia de pandillas criminales en la zona residencial. Asimismo, se halló que vivir con los padres hasta cierta edad crítica disminuye la probabilidad de consumo frecuente de marihuana en jóvenes. Estos resultados podrían ayudar a desarrollar estrategias y políticas públicas que ayuden a prevenir el consumo de marihuana y otras drogas desde edades tempranas.

Palabras clave: Cannabis; uso de la marihuana; trastornos relacionados con sustancias; Perú; poblaciones vulnerables.

\begin{abstract}
Marijuana is the most widely used illicit drug in the world, especially among young people. This study is relevant to policy makers because it expands the knowledge regarding drug use in vulnerable youth, allowing health authorities to reduce marijuana consumption via educational, family, and governmental strategies and policies. The objective of this study was to determine the prevalence of frequent marijuana consumption and its associated factors in young people before admission to juvenile detention centers in Peru. The data was taken from the 2016 National Population Census of the Youth Diagnostic and Rehabilitation Centers in Peru. The final sample was made up of 1,848 people with ages between 14 and 22 years old, with a median age of 17 (95.6\% males). The variable frequent marijuana consumption was defined as the use of marijuana at least once a week, prior to entering the center. The main factors associated with frequent marijuana use were male sex, running away from home before the age of 15 , physical abuse during childhood, having a family member who consumed alcohol or drugs frequently, and the presence of criminal gangs in the housing area. Additionally, it was found that living with parents up to a specific critical age decreases the probability of frequent use of marijuana in young people. These results could aid the development of strategies and public policies that help prevent the consumption of marijuana and other drugs from an early age.

Keywords: Cannabis; marijuana use; substance-related disorders; Peru; vulnerable populations.
\end{abstract}




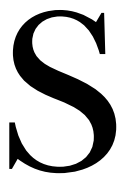

egún la Oficina de las Naciones Unidas contra la Droga y el Delito (UNODC, por sus siglas en inglés) y la Organización Mundial de la Salud (OMS), la marihuana es la sustancia ilícita más comúnmente consumida a nivel mundial, con aproximadamente 188 millones de consumidores en 2017 (Oficina de las Naciones Unidas contra la Droga y el Delito, 2019; Organización Mundial de la Salud, 2019). A nivel mundial, la prevalencia del consumo de marihuana oscila entre el 2,7 \% y el 4,9\%, y las tasas de consumo más altas se registran en Australia y América del Norte. En América del Sur, las tasas más altas de consumo de cannabis se encontraron en Brasil y Chile (Degenhardt, Ferrari y Hall, 2017; Degenhardt et al., 2013; Oficina de las Naciones Unidas contra la Droga y el Delito, 2015; Organización Mundial de la Salud, 2016). En Perú, la marihuana es también la droga ilícita más consumida. La tasa general de uso de marihuana en el último año se estima entre un 2-3 \% (Inter-American Drug Abuse Control Comission, 2019). Además, una encuesta nacional realizada en la población general encontró que el 47,1 \% de los consumidores de marihuana en Perú tienen signos de dependencia, similar a lo encontrado para los consumidores de cocaína (Comisión Nacional Para el Desarrollo y Vida sin Drogas, 2012).

El consumo de marihuana se ha asociado con efectos adversos agudos y a largo plazo. Los efectos agudos se desarrollan durante un corto período de tiempo después de consumir la sustancia e incluyen efectos psiquiátricos, como deterioro de la atención, discronometría, y psicosis; y síntomas físicos como hiperemia conjuntival, aumento del apetito, xerostomía, aumento de la presión arterial y aumento de la frecuencia cardíaca (Fonseca-Pedrero, Lucas-Molina, Pérez-Albéniz, Inchausti y Ortuño-Sierra, 2020; García Álvarez, Gomar, García-Portilla y Bobes, 2019; Karila et al., 2014; Levine, Clemenza, Rynn y Lieberman, 2017; Instituto Nacional sobre el Abuso de Drogas, 2019; Organización Mundial de la Salud, 2019). Los efectos a largo plazo incluyen deterioro psicológico, déficits cognitivos y consecuencias respiratorias (Guxens, Nebot, Ariza y Ochoa, 2007; Karila et al., 2014; Martínez-Torres et al., 2016; Organización Mundial de la Salud, 2019). Además, la dependencia al cannabis es responsable de una menor participación en actividades y relaciones académicas, menores ingresos, embarazos no planificados, enfermedades mentales, mayores tasas de desempleo y participación en otras conductas de riesgo (Khalsa y Baler, 2019; Oficina de las Naciones Unidas contra la Droga y el Delito, 2018). No obstante, el cannabis se ha asociado con algunos efectos beneficiosos; las afecciones más comunes por las que los médicos recomiendan el consumo de marihuana son dolor, náuseas y vómitos persistentes, mejora del sueño y ansiedad (Burggren, Shirazi, Ginder y London, 2019; Fiz, Durán, Capella, Carbonell y Farré, 2011).

La adolescencia ( 12 a 17 años) es un período de riesgo crítico para la iniciación del abuso de sustancias (Oficina de las Naciones Unidas contra la Droga y el Delito, 2018). Su fácil disponibilidad, junto con la percepción de un bajo riesgo de daño, hace que la marihuana sea la droga de elección en este grupo de edad (Comisión Nacional para el Desarrollo y Vida sin Drogas, 2012; Comisión Nacional para el Desarrollo y Vida sin Drogas, 2013; Oficina de las Naciones Unidas contra la Droga y el Delito, 2018). En Perú, la edad promedio de inicio del consumo de marihuana es de 14,4 años, y la prevalencia por año de consumo de marihuana entre los 12 y 17 años es del $2 \%$ (solo superada por el grupo de edad entre 19 y 25 años, con 2,1\%) (Comisión Nacional para el Desarrollo y Vida sin Drogas, 2012). El uso regular de marihuana durante la adolescencia es especialmente preocupante, ya que el uso en este grupo de edad se asocia con una mayor probabilidad de experimentar las consecuencias perjudiciales descritas anteriormente (Volkow, Baler, Compton y Weiss, 2014). Entre los adolescentes en los centros juveniles de diagnóstico y rehabilitación en Perú, se encontró que la prevalencia por año de consumo de marihuana es de aproximadamente 29,3\%, lo cual es más de diez veces superior a lo que se encontró para la población peruana en general (Comisión Nacional para el Desarrollo y Vida sin Drogas, 2013).

Los factores en la literatura científica que se han relacionado con el consumo de marihuana en adolescentes incluyen una variedad de factores sociales, interpersonales e individuales. Los factores sociales incluyen la urbanización y la disponibilidad de drogas en el entorno; los factores interpersonales incluyen la afiliación con compañeros que consumen marihuana y la presión de los pares; y los factores individuales incluyen género, nivel de estudio, percepción de riesgo, estrés percibido, autoestima, impulsividad y malestar emocional (Ameth et al., 2017; Casajuana et al., 2021; Rial et al., 2019; Zapata Roblyer, Betancourth y Grzywacz, 2015).

El objetivo de este trabajo es conocer la prevalencia, las características y los factores asociados al consumo de marihuana entre jóvenes de los centros juveniles de diagnóstico y rehabilitación en Perú. Aunque se dispone de literatura internacional sobre los factores asociados al consumo de marihuana en adolescentes, nuestra revisión no encontró estudios sobre el consumo de marihuana en jóvenes de centros juveniles de diagnóstico y rehabilitación.

\section{Materiales y métodos}

Este estudio tiene un diseño analítico transversal, basado en el análisis secundario de los datos obtenidos del Censo Nacional de Población en los Centros de Diagnóstico y Rehabilitación Juvenil en Perú, realizado entre marzo y abril de 2016 por el Instituto Nacional de Estadística e Informática (INEI), en coordinación con el Ministerio de Justicia. El censo incluyó a todos los y las jóvenes admitidos en los 10 centros juveniles de diagnóstico y rehabilitación ubica- 
dos en todo Perú (Oficina de las Naciones Unidas contra la Droga y el Delito, 2019). Considerando una prevalencia de consumo de marihuana de 2,5\% (Organización Mundial de la Salud, 2019) y un nivel de significancia de $95 \%$, estimamos el poder estadístico para dos escenarios de comparación de proporciones (depresión y consumo de tabaco) (Bahorik et al., 2018; Leatherdale, Ahmed y Kaiserman, 2006) en OpenEpi versión 3.01. Así, obtuvimos una potencia estadística superior al $90 \%$ en ambos escenarios.

La información se recopiló a través de un cuestionario presencial mediante una ficha censal impresa, y el encuestador registró las respuestas. La ficha censal incluyó una portada y cinco capítulos con 179 preguntas distribuidas en 16 páginas. Los capítulos de la encuesta fueron: condiciones sociales y familiares, situación del delito, condiciones de vida, papel de las instituciones y expectativas del recluso. Una prueba piloto se realizó para evaluar la operatividad, estructura, redacción y comprensión de las preguntas, y las alternativas de respuesta indicadas en la ficha censal. Como resultado, el número de preguntas se redujo de 213 a 179 para acortar la duración del cuestionario. Se capacitó a los coordinadores de los centros juveniles y los encuestadores del censo para garantizar la calidad de los datos obtenidos. También se les entregó un manual que sirvió de guía para una adecuada recolección de información por parte del encuestador (Instituto Nacional de Estadística e Informática, 2016).

La variable de resultado consumo frecuente de marihuana (CFM) se construyó a partir de tres preguntas de la sección de salud del cuestionario: la primera pregunta fue: «¿Usó drogas antes de ingresar al centro juvenil de diagnóstico y rehabilitación?». En caso de una respuesta afirmativa, el recluso respondería a: «¿Qué tipo de droga consumió?». Tercero, se les preguntó: «¿Con qué frecuencia consumía antes de ingresar al centro juvenil?». Se consideró que tenían la variable de resultado los participantes que respondieron «Sí» a la primera pregunta; «marihuana» a la segunda; $\mathrm{y}$ «a diario», «entre 2 y 6 veces por semana»o «semanalmente» a la tercera. De esta forma se pudieron evaluar los posibles factores asociados al consumo frecuente de marihuana en jóvenes antes de ingresar a los centros juveniles de diagnóstico y rehabilitación.

Las variables se dividieron en sociodemográficas y características familiares, morbilidades y hábitos nocivos. La variable región se dividió según el lugar de origen del recluso. Fueron clasificados como provenientes de Lima $\mathrm{u}$ otras regiones. El nivel de educación se clasificó en sin estudios, estudios primarios (terminados o no terminados) y estudios secundarios (terminados o no terminados). Se consideró trabajo infantil en quienes informaron haber trabajado a los 14 años o antes. La edad hasta la que el recluso vivió con la madre y el padre se clasificó en: nunca vivió, vivió hasta los 14 años y vivió más allá de los 14 . Además, las variables independientes fueron: sexo, edad, huir de casa antes de los 15 años, maltrato físico durante la niñez, haber tenido durante la niñez un familiar que consumía alcohol o drogas con frecuencia, presencia de pandillas en la zona donde vivía, discriminación o maltrato antes del ingreso, haber pertenecido a alguna pandilla delictiva, y reingreso al centro juvenil de diagnóstico y rehabilitación. La depresión, el trastorno por abuso de sustancias y el asma se evaluaron mediante dos preguntas. La primera pidió al encuestado que autoinformase si tenía o no la enfermedad. La segunda preguntó si un profesional sanitario le había diagnosticado esta enfermedad. Solo aquellos que respondieron afirmativamente a estas dos preguntas fueron considerados como portadores de la enfermedad. Las limitaciones permanentes en el aprendizaje y la concentración se evaluaron mediante autoinforme. Los hábitos nocivos previos al ingreso (consumo de bebidas alcohólicas, tabaco y edad de inicio) fueron autoinformados. El autoinforme de enfermedades ha sido utilizado en estudios previos realizados en poblaciones vulnerables (Feinstein et al., 1998; Salazar-De La Cuba, Ardiles-Paredes, Araujo-Castillo y Maguiña, 2019). Un estudio concluyó que la prevalencia de enfermedades autoinformadas era más sensible y específica que la incidencia (Oksanen et al., 2010).

La base de datos se descargó de https://observatorio. mininter.gob.pe/proyectos/censo-nacional-de-centros-juveniles en formato dbf y se transfirió a Stata 14.0 (Stata Corporation, College Station, Texas, EE. UU.) para su análisis. En el análisis univariado se describieron las frecuencias absolutas y relativas de cada variable de interés. Las variables numéricas se describieron a través de la mediana y el rango intercuartílico (RIQ), debido a su distribución anormal, previamente testeada con el test de Shapiro-Wilk. El análisis bivariado se realizó mediante la prueba de chi cuadrado de Pearson para las variables categóricas y la prueba $U$ de Mann-Whitney para las variables numéricas. La magnitud de la asociación se calculó mediante la Razón de Prevalencia Cruda (RPc) y la Razón de Prevalencia Ajustada (RPa) calculadas para cada variable. Se tuvieron en cuenta los criterios epidemiológicos y estadísticos para seleccionar las variables del modelo multivariable. Ambos modelos se estimaron empleando modelos lineales generalizados, familia de Poisson, función de enlace de logaritmo, con varianza robusta. Este modelo estadístico nos permite estudiar las asociaciones entre exposiciones y resultados binarios, mediante estimaciones puntuales y errores estándar comparables (Chen, Qian, Shi y Franklin, 2018). Esto nos permite determinar los riesgos relativos que, basado en el diseño de este estudio, se interpretarían como razones de prevalencia. El modelo multivariable se ajustó para todas las variables previamente seleccionadas y se ajustó por conglomerados conformados por los centros juveniles de diagnóstico y rehabilitación. Todos los análisis estadísticos se realizaron con un nivel de significancia menor a 0,05 y un intervalo de confianza del $95 \%$ (IC $95 \%$ ). Este tra- 
bajo fue aprobado por el comité de ética institucional de la Universidad Peruana de Ciencias Aplicadas (código de registro PI081-17).

\section{Resultados}

La población de los 10 centros juveniles de diagnóstico y rehabilitación fue de 2203. De estos, 58 fueron excluidos porque estuvieron ausentes durante la encuesta o entregaron formularios en blanco. De los 1965 restantes, 117 fueron excluidos porque no cumplieron con las variables de interés. Se incluyeron 1848 participantes, lo que representa el 91,3\% de la población total (ver Figura 1).

El CFM, como se definió anteriormente, tuvo una prevalencia del 32,8 \% ( $\mathrm{n}=606)$. El rango de edad fue de 14 a 22 años con una mediana de edad de 17 años (RIQ: 16-18). Además, el 95,29 \% $(\mathrm{n}=1761)$ de los encuestados eran hombres y el 67,26\% $(\mathrm{n}=1243)$ tenía estudios secundarios. Se encontró una asociación estadísticamente significativa (valor de $p<, 05$ ) entre el CFM y nacer en Lima, huir de casa antes de los 15 años, presencia del padre, maltrato físico durante la niñez, tener un familiar que consumía alcohol o drogas con frecuencia, presencia de pandillas criminales en el área de residencia, pertenecer a una pandilla criminal y reingreso al centro juvenil de diagnóstico y rehabilitación (ver Tabla 1). También se encontraron asociaciones estadísticamente significativas entre el CFM y un diagnóstico médico de trastorno por abuso de sustancias, consumo de bebidas alcohólicas y consumo de tabaco. También se encontró una asociación entre la edad de inicio del consumo de alcohol y tabaco y el CFM (ver Tabla 2).

Cuando se ajustó por todas las variables del modelo y los centros juveniles de diagnóstico y rehabilitación como clusters, se encontró que el sexo masculino (RPa: 1,31, 95
\% IC: 1,21-1,40), haber nacido en Lima (RPa: 1,64, $95 \%$ IC: 1,36-1,95), huir de casa antes de los 15 años (RPa: 1,28, $95 \%$ IC: 1,11-1,47), maltrato físico durante la niñez (RPa: 1,23, $95 \%$ IC: 1,07-1,40), tener un familiar que consumía alcohol con frecuencia (RPa: 1,08, $95 \%$ IC: 1,01-1,15), tener un familiar que consumía drogas (RPa: 1,19, $95 \%$ IC: 1,05-1,34), presencia de pandillas criminales en el área de residencia (RPa: 1,63, 95 \% IC: 1,46-1,82), reingreso al centro juvenil de diagnóstico y rehabilitación (RPa: 1,18, 95 \% IC: 1,02-1,38), diagnóstico médico de trastorno por abuso de sustancias (RPa: 1,38, 95 \% IC: 1,13-1,67) y consumo de bebidas alcohólicas (RPa: 1,40, 95 \% IC: 1,18-1,66) aumentó la probabilidad del CFM de una manera estadísticamente significativa. También encontramos que, en comparación con no haber vivido nunca con la madre, vivir con ella hasta los 14 años (RPa: 0,79, 95 \% IC: 0,64-0,95) o más (RPa: 0,83, 95 \% IC: 0,69-0,99) disminuyó la probabilidad de CFM. En el caso del padre, solo vivir con él más allá de los 14 años (RPa: 0,83, 95 \% IC: 0,73-0,93) disminuyó la probabilidad de CFM (ver Tabla 3).

\section{Discusión}

Según el Informe mundial sobre las drogas 2015, los hombres tienen tres veces más probabilidades que las mujeres de consumir marihuana (Oficina de las Naciones Unidas contra la Droga y el Delito, 2015). En nuestro país, el consumo de marihuana en los hombres es entre tres y diez veces superior al de las mujeres (Comisión Nacional para el Desarrollo y Vida sin Drogas, 2012). Esto también se observó en nuestro estudio, donde los participantes masculinos tenían 1,31 veces más probabilidades que las mujeres de consumir marihuana al menos una vez por semana. La prevalencia del consumo de marihuana entre los grupos de edad varía entre regiones y nivel socioeconómi-

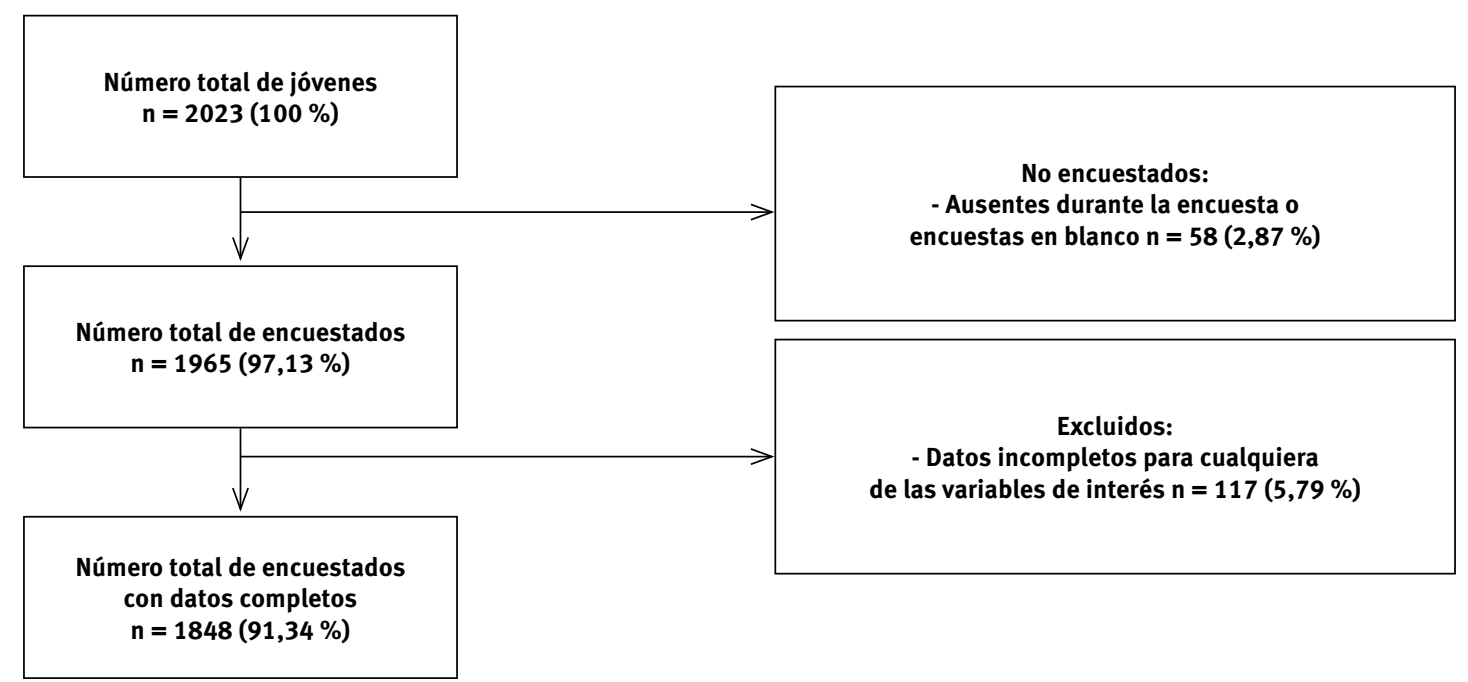

Figura 1. Diagrama de flujo. 
Ali Al-kassab-Córdova, Gonzalo Cornejo-Venegas, Nataly Gacharna-Madrigal,

Carlos Baquedano-Rojas, Guillermo Nicolás De La Borda Prazak, Christian R. Mejia

Tabla 1. Prevalencia de consumo frecuente de marihuana (CFM) en centros juveniles de diagnóstico y rehabilitación peruvianos según características sociodemográficas y familiares.

\begin{tabular}{|c|c|c|c|c|c|}
\hline Características & $\begin{array}{l}\text { Población del estudio } \\
(\mathrm{n}=1848) \\
\mathrm{n}(\%)\end{array}$ & $\begin{array}{c}\text { CFM }^{*} \dagger \\
(n=606)\end{array}$ & $\begin{array}{c}\text { Prevalencia } \\
\text { (\% fila) }\end{array}$ & IC $95 \%$ & valor $p$ \\
\hline Edad (RIC) & $17(16-18)$ & $17(16-18)$ & & & 0,127 \\
\hline \multicolumn{6}{|l|}{ Sexo } \\
\hline Hombre & $1761(95,29)$ & 579 & 32,88 & $30,72-35,11$ & \multirow{2}{*}{0,721} \\
\hline Mujer & $87(4,71)$ & 27 & 31,03 & $22,17-41,54$ & \\
\hline \multicolumn{6}{|l|}{ Región } \\
\hline Lima & $866(46,86)$ & 375 & 43,30 & $40,03-46,63$ & \multirow{2}{*}{$<0,001$} \\
\hline Otras regiones & $983(53,14)$ & 231 & 23,52 & $20,97-26,28$ & \\
\hline \multicolumn{6}{|l|}{ Nivel de estudios } \\
\hline Sin estudios & $22(1,19)$ & 6 & 27,27 & $12,54-49,50$ & \multirow{3}{*}{0,183} \\
\hline Primarios & $583(31,55)$ & 208 & 35,68 & $31,88-39,65$ & \\
\hline Secundarios & $1243(67,26)$ & 392 & 31,54 & $29,00-34,17$ & \\
\hline \multicolumn{6}{|l|}{ Trabajo infantil } \\
\hline Sí & $932(50,43)$ & 298 & 31,97 & $29,05-35,04$ & \multirow{2}{*}{0,450} \\
\hline No & $916(49,57)$ & 308 & 33,62 & $30,63-36,75$ & \\
\hline \multicolumn{6}{|c|}{ Huyó de casa antes de la edad de 15 años } \\
\hline Sí & $717(38,80)$ & 307 & 42,82 & $39,23-46,47$ & \multirow{2}{*}{$<0,001$} \\
\hline No & $1131(61,20)$ & 299 & 26,44 & $23,94-29,08$ & \\
\hline \multicolumn{6}{|c|}{ Edad hasta la cual convivió con la madre } \\
\hline Nunca convivió & $58(3,14)$ & 26 & 44,83 & $32,51-57,80$ & \multirow{3}{*}{0,030} \\
\hline$\leq 14$ años & $534(28,90)$ & 189 & 35,39 & $31,44-39,55$ & \\
\hline$>14$ años & $1256(67,97)$ & 391 & 31,13 & $28,62-33,74$ & \\
\hline \multicolumn{6}{|c|}{ Edad hasta la cual convivió con el padre } \\
\hline Nunca convivió & $280(15,15)$ & 114 & 40,71 & $35,09-46,58$ & \multirow{3}{*}{$<0,001$} \\
\hline$\leq 14$ años & $724(39,18)$ & 251 & 34,67 & $31,28-38,21$ & \\
\hline$>14$ años & $844(45,67)$ & 241 & 28,55 & $25,60-31,70$ & \\
\hline \multicolumn{6}{|c|}{ Maltrato infantil durante la niñez } \\
\hline Sí & $866(46,86)$ & 329 & 37,99 & $34,81-41,27$ & \multirow{2}{*}{$<0,001$} \\
\hline No & $982(53,14)$ & 277 & 28,21 & $25,47-31,10$ & \\
\hline \multicolumn{6}{|c|}{ Haber tenido durante la niñez un familiar que consumía alcohol con frecuencia } \\
\hline Sí & $580(31,39)$ & 234 & 40,34 & $36,41-44,39$ & \multirow{2}{*}{$<0,001$} \\
\hline No & $1268(68,61)$ & 372 & 29,34 & $26,89-31,90$ & \\
\hline \multicolumn{6}{|c|}{ Haber tenido durante la niñez un familiar que consumía drogas } \\
\hline Sí & $81(4,38)$ & 49 & 60,49 & $49,43-70,57$ & \multirow{2}{*}{$<0,001$} \\
\hline No & $1767(95,62)$ & 557 & 31,52 & $29,39-33,72$ & \\
\hline \multicolumn{6}{|c|}{ Presencia de pandillas en la zona donde vivía } \\
\hline Sí & $975(52,76)$ & 426 & 43,69 & $40,60-46,83$ & \multirow{2}{*}{$<0,001$} \\
\hline No & $873(47,24)$ & 180 & 20,62 & $18,06-23,43$ & \\
\hline \multicolumn{6}{|c|}{ Discriminación o abuso antes del ingreso } \\
\hline Sí & $166(8,98)$ & 66 & 39,76 & $32,57-47,41$ & 0015 \\
\hline No & $1682(91,02)$ & 540 & 32,10 & $29,91-34,37$ & 0,043 \\
\hline Pertenencia a algu & tiva & & & & \\
\hline Sí & $337(18,24)$ & 154 & 45,70 & $40,43-51,05$ & $<001$ \\
\hline No & $1511(81,76)$ & 452 & 29,91 & $27,65-32,27$ & 0,001 \\
\hline Readmisión a un c & diagnóstico y rehabilitac & & & & \\
\hline Sí & $253(13,69)$ & 112 & 44,27 & $38,24-50,46$ & $<0001$ \\
\hline No & $1595(86,31)$ & 494 & 30,97 & $28,74-33,28$ & 10,001 \\
\hline
\end{tabular}

Nota. * Se utilizaron tests de chi cuadrado para las variables categóricas y la prueba de U de Mann-Whitney para las variables numéricas.

†CFM: Consumo frecuente de marihuana, definido como consumo al menos una vez por semana. 
Tabla 2. Prevalencia de consumo frecuente de marihuana (CFM) en centros juveniles de diagnóstico y rehabilitación peruvianos según morbilidades y hábitos nocivos.

\begin{tabular}{|c|c|c|c|c|c|}
\hline Características & $\begin{array}{l}\text { Población del estudio } \\
\qquad \begin{array}{c}(n=1848) \\
n(\%)\end{array}\end{array}$ & $\begin{array}{c}\text { CFM*t } \\
(n=606)\end{array}$ & $\begin{array}{l}\text { Prevalencia } \\
\text { (\% fila) }\end{array}$ & IC $95 \%$ & Valor $p$ \\
\hline \multicolumn{6}{|l|}{ Morbilidades $¥$} \\
\hline \multicolumn{6}{|l|}{ Depresión } \\
\hline Sí & $69(3,73)$ & 31 & 44,93 & $33,58-56,82$ & \multirow{2}{*}{0,029} \\
\hline No & $1779(96,27)$ & 575 & 32,32 & $30,18-34,53$ & \\
\hline \multicolumn{6}{|l|}{ Trastorno por uso de sustancias } \\
\hline Sí & $64(3,46)$ & 39 & 60,94 & $48,45-72,13$ & \multirow{2}{*}{$<0,001$} \\
\hline No & $1784(96,54)$ & 567 & 31,78 & $29,66-33,98$ & \\
\hline \multicolumn{6}{|l|}{ Asma } \\
\hline Sí & $105(5,68)$ & 42 & 40,00 & $31,04-49,67$ & \multirow{2}{*}{0,105} \\
\hline No & $1743(94,32)$ & 564 & 32,36 & $30,19-34,59$ & \\
\hline \multicolumn{6}{|c|}{ Limitaciones permanentes en la comprensión o el aprendizaje } \\
\hline Sí & $289(15,64)$ & 106 & 36,68 & $31,30-42,40$ & \multirow{2}{*}{0,126} \\
\hline No & $1559(84,36)$ & 500 & 32,07 & $29,79-34,43$ & \\
\hline \multicolumn{6}{|l|}{ Hábitos nocivost } \\
\hline Edad de inicio del uso de marihuana (RIC) & & $14(13-15)$ & & & $<0,001$ \\
\hline \multicolumn{6}{|l|}{ Consumo de bebidas alcohólicas } \\
\hline Sí & $1507(81,55)$ & 535 & 35,50 & $33,12-37,95$ & \multirow{2}{*}{$<0,001$} \\
\hline No & $341(18,45)$ & 71 & 20,82 & $16,83-25,46$ & \\
\hline Edad de inicio de consumo de alcohol (RIC) & & $14(13-15)$ & & & $<0,001$ \\
\hline \multicolumn{6}{|l|}{ Uso de tabaco } \\
\hline Sí & $925(50,05)$ & 344 & 37,19 & $34,12-40,35$ & \multirow{2}{*}{$<0,001$} \\
\hline No & $923(49,95)$ & 262 & 28,39 & $25,56-31,38$ & \\
\hline Edad de inicio del uso de tabaco (RIC) & & $14(13-15)$ & & & $<0,001$ \\
\hline
\end{tabular}

Nota. * Se utilizaron tests de chi cuadrado para las variables categóricas y la prueba de U de Mann-Whitney para las variables numéricas.

† CFM: Consumo frecuente de marihuana, definido como consumo al menos una vez por semana.

‡ Enfermedades autoinformadas diagnosticadas por profesionales sanitarios.

† Uso antes de la admisión al centro juvenil de diagnóstico y rehabilitación.

co. Según la Organización Mundial de la Salud, en los Estados Unidos el consumo de marihuana alcanza su punto máximo a principios de la edad de veinte años y disminuye a finales de los veinte. En Brasil, la prevalencia de consumo es más alta entre la población adolescente (Organización Mundial de la Salud, 2016). En este estudio, los sujetos tenían entre 14 y 22 años, un grupo de edad ideal para estudiar el consumo de marihuana porque, como se ha explicado con anterioridad, la mayor prevalencia se encuentra entre los adolescentes y los adultos jóvenes.

Varios estudios han demostrado que las interacciones interpersonales influyen en la probabilidad de consumo de marihuana. En Perú, el entorno de iniciación más común para el consumo de marihuana es entre los grupos de vecinos, que representan el 57,9 \% de los entornos de iniciación al consumo (Comisión Nacional para el Desarrollo y Vida sin Drogas, 2012). Además, un estudio realizado en Carolina del Norte mostró una correlación positiva entre la pertenencia a pandillas y la frecuencia del consumo de marihuana. El mismo estudio también mostró una mayor probabilidad de consumo en la adolescencia temprana en comparación con aquellos sin pertenencia a una pandilla (Wechsberg et al., 2015). Estos resultados son consistentes con los nuestros porque hemos descrito un aumento del $63 \%$ de la probabilidad de tener CFM entre los que viven en un vecindario con la presencia de pandillas. Otro estudio encontró que los adolescentes cuyos compañeros estaban involucrados en el consumo de drogas, incluida la marihuana, tenían casi seis veces más posibilidades de consumir cannabis, muy probablemente debido a la presión de los compañeros (Mehanović et al., 2020).

Se ha informado que la situación familiar influye sobre el consumo de marihuana, y esto es consistente con los resultados de este estudio. Tener padres que consumen alcohol y drogas aumenta la probabilidad de CFM en 1,08 y 1,19 veces, respectivamente. Se ha descrito que la estrechez de los lazos entre los jóvenes y sus padres reduce el consumo de marihuana y otras sustancias (Kosterman, Hawkins, Guo, Catalano y Abbott, 2000; Velleman, Templeton y Copello, 2005). Además, una revisión sistemática encontró 
Ali Al-kassab-Córdova, Gonzalo Cornejo-Venegas, Nataly Gacharna-Madrigal,

Carlos Baquedano-Rojas, Guillermo Nicolás De La Borda Prazak, Christian R. Mejia

Tabla 3. Factores asociados con el consumo frecuente de marihuana (CFM) en los centros juveniles de diagnóstico y rehabilitación peruvianos.

\begin{tabular}{|c|c|c|c|c|c|c|}
\hline & \multicolumn{3}{|c|}{ Análisis crudo ${ }^{\mathrm{a}}$} & \multicolumn{3}{|c|}{ Modelo ajustado $^{b}$} \\
\hline & $\mathrm{RPc}^{*}$ & Valor $\mathbf{p}$ & IC $95 \%$ & RPa‡ & Valor $\mathbf{p}$ & IC $95 \%$ \\
\hline Edad & 1,02 & 0,370 & $0,97-1,06$ & 0,96 & 0,034 & $0,92-0,99$ \\
\hline \multicolumn{7}{|l|}{ Sexo } \\
\hline Hombre & 1,06 & 0,724 & $0,76-1,45$ & 1,31 & $<0,001$ & $1,21-1,40$ \\
\hline Mujer & Ref & Ref & Ref & Ref & Ref & Ref \\
\hline \multicolumn{7}{|l|}{ Región } \\
\hline Lima & 1,84 & $<0,001$ & $1,60-2,10$ & 1,64 & $<0,001$ & $1,36-1,95$ \\
\hline Otras regiones & Ref & Ref & Ref & Ref & Ref & Ref \\
\hline \multicolumn{7}{|c|}{ Huyó de casa antes de la edad de 15 años } \\
\hline Sí & 1,62 & $<0,001$ & $1,42-1,84$ & 1,28 & 0,001 & $1,11-1,47$ \\
\hline No & Ref & Ref & Ref & Ref & Ref & Ref \\
\hline \multicolumn{7}{|c|}{ Edad hasta la cual convivió con la madre } \\
\hline Nunca convivió & Ref & Ref & Ref & Ref & Ref & Ref \\
\hline$\leq 14$ años & 0,79 & 0,132 & $0,58-1,07$ & 0,79 & 0,014 & $0,64-0,95$ \\
\hline$>14$ años & 0,69 & 0,016 & $0,51-0,93$ & 0,83 & 0,049 & $0,69-0,99$ \\
\hline \multicolumn{7}{|c|}{ Edad hasta la cual convivió con el padre } \\
\hline Nunca convivió & Ref & Ref & Ref & Ref & Ref & Ref \\
\hline$\leq 14$ años & 0,85 & 0,069 & $0,71-1,01$ & 0,91 & 0,337 & $0,74-1,10$ \\
\hline$>14$ años & 0,70 & 0,000 & $0,58-0,83$ & 0,83 & 0,002 & $0,73-0,93$ \\
\hline \multicolumn{7}{|c|}{ Maltrato infantil durante la niñez } \\
\hline Sí & 1,35 & $<0,001$ & $1,18-1,53$ & 1,23 & 0,002 & $1,07-1,40$ \\
\hline No & Ref & Ref & Ref & Ref & Ref & Ref \\
\hline \multicolumn{7}{|c|}{ Haber tenido durante la niñez un familiar que consumía alcohol con frecuencia } \\
\hline Sí & 1,38 & $<0,001$ & $1,20-1,56$ & 1,08 & 0,016 & $1,01-1,15$ \\
\hline No & Ref & Ref & Ref & Ref & Ref & Ref \\
\hline \multicolumn{7}{|c|}{ Haber tenido durante la niñez un familiar que consumía drogas } \\
\hline Sí & 1,92 & $<0,001$ & $1,58-2,31$ & 1,19 & 0,005 & $1,05-1,34$ \\
\hline No & Ref & Ref & Ref & Ref & Ref & Ref \\
\hline \multicolumn{7}{|c|}{ Presencia de pandillas en la zona donde vivía } \\
\hline Sí & 2,12 & $<0,001$ & $1,82-2,45$ & 1,63 & $<0,001$ & $1,46-1,82$ \\
\hline No & Ref & Ref & Ref & Ref & Ref & Ref \\
\hline \multicolumn{7}{|c|}{ Discriminación o abuso antes del ingreso } \\
\hline Sí & 1,24 & 0,036 & $1,01-1,51$ & & & \\
\hline No & Ref & Ref & Ref & & & \\
\hline \multicolumn{7}{|c|}{ Pertenencia a alguna pandilla delictiva antes del ingreso } \\
\hline Sí & 1,53 & $<0,001$ & $1,32-1,75$ & & & \\
\hline No & Ref & Ref & Ref & & & \\
\hline \multicolumn{7}{|c|}{ Readmisión a un centro juvenil de diagnóstico y rehabilitación } \\
\hline Sí & 1,43 & $<0,001$ & $1,22-1,67$ & 1,18 & 0,027 & $1,02-1,38$ \\
\hline No & Ref & Ref & Ref & Ref & Ref & Ref \\
\hline \multicolumn{7}{|l|}{ Depresión } \\
\hline Sí & 1,22 & 0,019 & $1,03-1,44$ & & & \\
\hline No & Ref & Ref & Ref & & & \\
\hline \multicolumn{7}{|c|}{ Trastorno por uso de sustancias } \\
\hline Sí & 1,92 & $<0,001$ & $1,55-2,35$ & 1,38 & 0,001 & $1,13-1,67$ \\
\hline No & Ref & Ref & Ref & Ref & Ref & Ref \\
\hline \multicolumn{7}{|c|}{ Consumo de bebidas alcohólicas } \\
\hline Sí & 1,71 & $<0,001$ & $1,37-2,12$ & 1,40 & $<0,001$ & $1,18-1,66$ \\
\hline No & Ref & Ref & Ref & Ref & Ref & Ref \\
\hline \multicolumn{7}{|l|}{ Uso de tabaco } \\
\hline Sí & 1,31 & $<0,001$ & $1,14-1,49$ & & & \\
\hline No & Ref & Ref & Ref & & & \\
\hline
\end{tabular}

Nota. ${ }^{\text {a }}$ Regresión de Poisson con varianza robusta.

${ }^{\mathrm{b}}$ Regresión de Poisson con varianza robusta ajustada por las variables seleccionadas, y ajustando los centros juveniles de diagnóstico y rehabilitación como conglomerados.

*RPc: Razón de prevalencia cruda.

‡ RPa: Razón de prevalencia ajustada. 
que tener una familia monoparental, además de una relación problemática con los padres, son factores de riesgo importantes para el consumo de marihuana en los jóvenes (Guxens et al., 2007). También se ha demostrado que haber sufrido maltrato infantil durante la niñez es un factor de riesgo para el consumo de marihuana, como lo demuestra un estudio realizado en adultos jóvenes de México, utilizando la Encuesta de Cohesión Social para la Prevención de la Violencia y la Delincuencia. Asimismo, otros estudios también informaron la misma asociación (Hornor, 2010; Hser, Longshore y Anglin, 2007; Huang et al., 2011; Vega-Cauich, Heredia y García, 2018). Nuestro estudio encontró que huir de casa antes de los 15 años tenía 1,28 más probabilidades de CFM, en coherencia con estudios anteriores realizados en adolescentes sin hogar y que huyeron de sus casas, donde se encontró que la prevalencia de consumo de marihuana fue mucho mayor en este grupo en comparación con la población en general, independientemente de sus motivos por huir (Rew, Taylor-Seehafer y Fitzgerald, 2001; Tyler, Gervais y Davidson, 2013; Wang, Chen, Lew-Ting, Chen y Chen, 2010). En un estudio sobre la adolescencia temprana se encontró una asociación moderada entre tener un hermano con consumo de alcohol o de marihuana durante la infancia y el consumo de marihuana en el último año (Heerde, Bailey, Toumbourou y Catalano, 2019; Terry-McElrath, O’Malley y Johnston, 2013). Tener padres con una actitud positiva hacia el consumo de drogas aumentó 1,35 veces la probabilidad de uso de sustancias entre los jóvenes (Heerde et al., 2019; Terry-McElrath et al., 2013). Asimismo, tener padres con una actitud positiva hacia el consumo de alcohol y de tabaco aumentó cinco veces la probabilidad de consumo de marihuana (Mehanović et al., 2020).

Este estudio describió que el consumo de alcohol aumenta 1,40 veces la probabilidad de CFM. Además, encontramos una gran prevalencia de tabaquismo $(50,05 \%)$ en nuestra población, a diferencia de la baja prevalencia de tabaquismo en la población general de Perú (Organización Mundial de la Salud, 2016). Un estudio de 2019 encontró que el consumo de alcohol o cigarrillos aumentaba la probabilidad de uso simultáneo de marihuana. Cabe señalar que este estudio encontró que el consumo de marihuana también aumentó la probabilidad de consumo de alcohol y/o cigarrillos (Roche et al., 2019). Además, un estudio poblacional realizado en Canadá encontró que los adolescentes que habían consumido tanto alcohol como tabaco tenían 188 veces más probabilidades de haber probado la marihuana (Leatherdale, Hammond y Ahmed, 2008). Estos estudios son consistentes con los nuestros, ya que encontramos que el consumo de alcohol aumenta 1,40 veces la probabilidad de CFM. Un estudio en el último año de secundaria encontró que el uso simultáneo de alcohol y marihuana tenía un fuerte vínculo con la dependencia percibida (Mehanovič et al., 2020), lo que apoya nuestros hallazgos de una asociación entre el trastorno por abuso de sustancias autoinformado y el consumo de marihuana. También se ha encontrado que una edad más temprana de inicio del consumo de alcohol y tabaco aumenta la prevalencia de CFM, en coherencia con estudios anteriores en poblaciones encarceladas (Racz et al., 2016). El inicio temprano del uso de sustancias se ha asociado con el policonsumo de drogas, un mayor riesgo de abuso de sustancias y conductas delictivas más tarde en la vida (Gordon, Kinlock y Battjes, 2004; Moss, Chen y Yi, 2014). Esta asociación es particularmente fuerte en adultos jóvenes encarcelados (Harrison, Ramo, Hall, Estrada-Gonzales y Tolou-Shams, 2019). Por tanto, los jóvenes involucrados en el sistema penal tienen mayor riesgo de sufrir estos trastornos y deberían ser merecedores de evaluación y más intervenciones.

Encontramos que la readmisión en un centro juvenil de diagnóstico y rehabilitación aumenta 1,18 veces la probabilidad de CFM en comparación con aquellos que no fueron readmitidos. Esto se correlaciona bien con los estudios que indican que el uso de drogas, en general, está asociado con una reincidencia más rápida (Aalsma et al., 2015). Esto indica que el consumo de drogas, en este caso marihuana, podría ser un factor importante para el reingreso al centro juvenil de diagnóstico y rehabilitación. Una posible explicación de esto podría ser que fueron detenidos por su uso ilegal de marihuana, o porque el uso de drogas los provocó a cometer un acto ilegal.

Este estudio, debido a la gran cobertura de la población de los centros juveniles de diagnóstico y rehabilitación de menores, permite extrapolar a nivel de Perú los datos estadísticos encontrados respecto a este grupo poblacional. Los hallazgos de esta investigación deben interpretarse teniendo en cuenta sus limitaciones. Debido a que la variable CFM se generó a partir de preguntas del cuestionario, representa solo una aproximación del patrón de consumo de marihuana, ya que no ha sido validada. Es importante considerar que algunos de los datos obtenidos se refieren a hechos ocurridos en la infancia, por lo que puede haber sesgo de memoria. Las enfermedades analizadas fueron autoinformes de los diagnósticos por parte de profesionales sanitarios, por lo que están sujetos a sesgo de información. El uso de una entrevista presencial para recopilar información sobre conductas de riesgo produce un sesgo de deseabilidad. Por tratarse de un estudio transversal, carece de temporalidad y es imposible determinar la causalidad. Sugerimos el desarrollo de estudios longitudinales con el objetivo de evaluar el consumo de marihuana como un posible factor de riesgo para ingreso a los centros juveniles de diagnóstico y rehabilitación de menores. Además, recomendamos evaluar los factores de riesgo en esta población a través de censos en otros países.

Este estudio encontró importantes factores sociodemográficos y experiencias vitales asociados con el CFM en jóvenes peruanos antes de su ingreso a centros juveniles de 
diagnóstico y rehabilitación. Estos resultados son relevantes para los legisladores porque amplían el conocimiento sobre el consumo de drogas en jóvenes vulnerables, como los que viven en vecindarios con presencia de pandillas y en familias monoparentales. Esto permite a las autoridades sanitarias reducir la prevalencia de factores asociados con el uso más frecuente de marihuana a través de estrategias y políticas educativas, familiares y gubernamentales. A nivel educativo, se podrían diseñar programas que promuevan estilos de vida más saludables, esclarezcan los peligros de la marihuana y otras drogas, o permitan testimonios de exusuarios de drogas. A nivel familiar, los resultados podrían permitir a los padres estar más atentos a sus hijos y ser conscientes de qué factores podrían llevar a sus hijos a consumir marihuana u otras drogas. A nivel gubernamental, el gobierno podría hacer anuncios televisivos o radiofónicos para educar a la población sobre el uso de drogas, o también podría formular políticas que ayuden a reducir la frecuencia de uso, como agregar más seguridad alrededor de las comunidades para ayudar a reducir el uso de marihuana en vecindarios vulnerables.

\section{Agradecimientos}

El artículo fue financiado por la Dirección de Investigación de la Universidad Peruana de Ciencias Aplicadas (A-078-2020).

Contribuciones de los autores: AA y CM participaron en la concepción y el diseño del estudio. AA realizó la recopilación y el análisis de datos. Todos los autores participaron en la interpretación de los resultados, redacción del manuscrito, revisión crítica del manuscrito y aprobación final del manuscrito.

\section{Conflicto de intereses}

Los autores declaran la inexistencia de conflicto de interés respecto de la publicación de este artículo.

\section{Referencias}

Aalsma, M. C., White, L. M., Lau, K. S., Perkins, A., Monahan, P. y Grisso, T. (2015). Behavioral health care needs, detention-based care, and criminal recidivism at community reentry from Juvenile detention: A multisite survival curve analysis. American Journal of Public Health, 105, 1372-1378. doi:10.2105/AJPH.2014.302529.

Ameth, J., Bustos, M. N., Fregoso, D. A., Fleiz, C., Gutiérrez, M. D., Amador, N. G. y Medina-Mora, M. E. (2017). Contextual factors associated with marijuana use in school population. Salud Mental, 40, 93-102. doi:10.17711/ SM.0185-3325.2017.012.

Bahorik, A. L., Sterling, S. A., Campbell, C. I., Weisner, C., Ramo, D. y Satre, D. D. (2018). Medical and non-med- ical marijuana use in depression: Longitudinal associations with suicidal ideation, everyday functioning, and psychiatry service utilization. Journal of Affective Disorders, 241, 8-14. doi:10.1016/j.jad.2018.05.065.

Burggren, A. C., Shirazi, A., Ginder, N. y London, E. D. (2019). Cannabis effects on brain structure, function and cognition: Considerations for medical uses on brain structure, function and cognition: Considerations for medical uses of cannabis and its derivatives. The American Journal of Drug and Alcohol Abuse, 45, 563-579. doi:10 $.1080 / 00952990.2019 .1634086$.

Casajuana, C., López-Pelayo, H., Oliveras, C., Colom, J., Gual, A. y Balcells-Oliveró, M. M. (2021). The relationship between motivations for cannabis consumption and problematic use. Adicciones, 33, 31-42. doi:10.20882/ adicciones. 1221.

Chen, W., Qian, L., Shi, J. y Franklin, M. (2018). Comparing performance between log-binomial and robust Poisson regression models for estimating risk ratios under model misspecification. BMC Medical Research Methodology, 18, 63. doi:10.1186/s12874-018-0519-5.

Comisión Nacional para el Desarrollo y Vida sin Drogas - DEVIDA. (2013). I Encuesta Nacional Sobre el Consumo de Drogas en Adolescentes Infractores del Poder Judicial. Recuperado de https://www.devida.gob.pe/ documents/20182/97548/I-Encuesta-Nacional-de-Consumo-de-Drogas-en-Adolescentes-Infractores-del-Poder-Judicial-2013.pdf/eccd674d-e0b5-46e0-a4ad$29 \mathrm{c} 5431968 \mathrm{c} 0$.

Comisión Nacional para el Desarrollo y Vida sin Drogas - DEVIDA. (2012). Informe Ejecutivo, Encuesta Nacional Consumo de Drogas. Recuperado de https://www. devida.gob.pe/documents/20182/97792/Informe-Ejecutivo-Encuesta-Nacional-sobre-Consumo-de-Drogas-en-Poblaci\%C3\%B3n-General-Per\%C3\%BA-2010. pdf/529e8f42-3641-4b5d-a787-54b626de0742.

Degenhardt, L., Ferrari, A. J. y Hall, W. D. (2017). The global epidemiology and disease burden of cannabis use and dependence. Handbook of cannabis and related pathologies: Biology, pharmacology, diagnosis, and treatment. (pp. 89-100). doi:10.1371/journal.pone.0165221.

Degenhardt, L., Ferrari, A. J., Calabria, B., Hall, W. H., Normal, R. E., McGrath, J. y Vos, T. (2013). The global epidemiology and contribution of cannabis use and dependence to the global burden of disease: Results from the GBD 2010 study. PloS One, 8, e76635. doi:10.1371/ journal.pone.0076635.

Feinstein, R. A., Lampkin, A., Lorish, C. D., Klerman, L. V., Maisiak, R. y Oh, M. K. (1998). Medical status of adolescents at time of admission to a juvenile detention center. The Journal of Adolescent Health, 22, 190-196. doi:10.1016/ S1054-139X(97)00169-9.

Fiz, J., Durán, M., Capellà, D., Carbonell, J. y Farré, M. (2011). Cannabis use in patients with fibromyalgia: Ef- 
fect on symptoms relief and health-related quality of life. PloS One, 6, e18440. doi:10.1371/journal.pone.0018440. Fonseca-Pedrero, E., Lucas-Molina, B., Pérez-Albéniz, A., Inchausti, F. y Ortuño-Sierra, J. (2020). Psychotic-like experiences and cannabis use in adolescents from the general population. Adicciones, 32, 41-51. doi:10.20882/ adicciones.1149.

García Álvarez, L., Gomar, J. J., García-Portilla, M. P. y Bobes, J. (2019). Cannabis use and cognitive impairment in schizophrenia and first-episode psychosis. Adicciones, 31, 89-94. doi:10.20882/adicciones.1328.

Gordon, M. S., Kinlock, T. W. y Battjes, R. J. (2004). Correlates of early substance use and crime among adolescents entering outpatient substance abuse treatment. American Journal of Drug and Alcohol Abuse, 30, 39-59. doi:10.1081/ADA-120029865.

Guxens M., Nebot M., Ariza C. y Ochoa, D. (2007). Factores asociados al inicio del consumo de cannabis: Una revisión sistemática de estudios de cohortes. Gaceta Sanitaria, 21, 252-260. doi:10.1157/13106812.

Harrison, A., Ramo, D., Hall, S. M., Estrada-Gonzalez, V. y Tolou-Shams, M. (2019). Cigarette smoking, mental health, and other substance use among court-involved youth. Substance Use E Misuse, 55, 572-581. doi:10.1080/ 10826084.2019.1691593.

Heerde, J. A., Bailey, J. A., Toumbourou, J. W. y Catalano, R. F. (2019). Longitudinal associations between the adolescent family environment and young adult substance use in Australia and the United States. Frontiers in Psychiatry, 10, 821. doi:10.3389/fpsyt.2019.00821.

Hornor, G. (2010). Child sexual abuse: Consequences and implications. Journal of Pediatric Health Care, 24, 358-364. doi:10.1016/j.pedhc.2009.07.003.

Hser, Y. I., Longshore, D. y Anglin, M. D. (2007). The life course perspective on drug use: A conceptual framework for understanding drug use trajectories. Evaluation Review, 31, 515-547. doi:10.1177/0193841X07307316.

Huang, S., Trapido, E., Fleming, L., Arheart, K., Crandall, L., French, M. y Prado, G. (2011). The long-term effects of childhood maltreatment experiences on subsequent illicit drug use and drug-related problems in young adulthood. Addictive Behaviors, 36, 95-102. doi:10.1016/j. addbeh.2010.09.001.

Instituto Nacional de Estadística e Informática (2016). Ficha Técnica del Censo Nacional de Población en los Centros Juveniles de Diagnóstico y Rehabilitación, 2016. Recuperado de https://www.inei.gob.pe/media/MenuRecursivo/censos/cejuv2016/CEJUV_01-01_Ficha_Tecnica.pdf.

Instituto Nacional de Estadística e Informática (2016). Informe Final del Censo Nacional de Población en los Centros Juveniles de Diagnóstico y Rehabilitación, 2016. Recuperado de https://www.inei.gob.pe/media/MenuRecursivo/censos/cejuv2016/Informe_Final_Juveniles.pdf.
Instituto Nacional sobre el Abuso de Drogas. (2020). Marijuana. National Institutes of Health. Recuperado de https://www.drugabuse.gov/drugs-abuse/marijuana.

Inter-American Drug Abuse Control Commission. (2019). Report on drug consumption in the Americas 2019. Recuperado de http:/ / www.cicad.oas.org/oid/Report $\% 20$ on $\% 20$ Drug $\% 20$ Use $\% 20$ in $\% 20$ the $\% 20$ Americas $\% 20$ 2019.pdf.

Karila, L., Roux, P., Rolland, B., Benyamina, A., Reynaud, M., Aubin, H. J. y Lançon, C. (2014). Acute and longterm effects of cannabis use: A review. Current Pharmaceutical Design, 20, 4112-4118. doi:10.2174/1381612811 3199990620 .

Khalsa, J. y Baler, R. (2019). Medical consequences of cannabis use. Cannabis Use Disorders (pp. 157-167). doi:10.1007/978-3-319-90365-1_15.

Kosterman, R., Hawkins, J. D., Guo, J., Catalano, R. F. y Abbott, R. D. (2000). The dynamics of alcohol and marijuana initiation: Patterns and predictors of first use in adolescence. American Journal of Public Health, 90, 360366. doi:10.2105/ajph.90.3.360.

Leatherdale, S. T., Ahmed, R. y Kaiserman, M. (2006). Marijuana use by tobacco smokers and nonsmokers: Who is smoking what? Canadian Medical Association Journal, 174, 1399. doi:10.1503/cmaj.051614.

Leatherdale, S. T., Hammond, D. y Ahmed, R. (2008). Alcohol, marijuana, and tobacco use patterns among youth in Canada. Cancer Causes Control, 19, 361-369. doi:10.1007/s10552-007-9095-4.

Levine, A., Clemenza, K., Rynn, M. y Lieberman, J. (2017). Evidence for the risks and consequences of adolescent cannabis exposure. Journal of the American Academy of Child and Adolescent Psychiatry, 56, 214-225. doi:10.1016/j. jaac.2016.12.014.

Martínez-Torres, J., Arias-Coronel, F., Rodelo-Vega, A., Jaraba-Toro, N., Meza-Castellanos, L., Contreras-Jáuregui, M.,...Villamizar-Carrillo, D. (2016). Prevalencia y factores asociados al consumo de marihuana en estudiantes de 18 a 25 años de una universidad pública, Colombia. Universidad y Salud, 18, 525-531. doi:10.22267/ rus. 161803.57.

Mehanović, E., Virk, H. K., Akanidomo, I., Pwajok, J., Prichard, G., Van-der-Kreeft, P. y Di Marco, V. (2020). Correlates of cannabis and other illicit drugs use among secondary school adolescents in Nigeria. Drug and Alcohol Dependence, 206, 107457. doi:10.1016/j.drugalcdep.2019.04.028.

Moss, H. B., Chen, C. M. y Yi, H. Y. (2014). Early adolescent patterns of alcohol, cigarettes, and marijuana polysubstance use and young adult substance use outcomes in a nationally representative sample. Drug and Alcohol Dependence, 136, 51-62. doi:10.1016/j.addbeh.2015.06.041.

Oficina de las Naciones Unidas contra la Droga y el Delito (2015). Informe mundial sobre las drogas 2015. Recupe- 
rado de https://www.unodc.org/documents/wdr2015/ World_Drug_Report_2015.pdf.

Oficina de las Naciones Unidas contra la Droga y el Delito (2019). Resumen ejecutivo del Informe mundial sobre las drogas 2019. Recuperado de https://wdr.unodc. org/wdr2019/prelaunch/WDR19_Booklet_1_EXECUTIVE_SUMMARY.pdf.

Oficina de las Naciones Unidas contra la Droga y el Delito. Informe mundial sobre las drogas (2018). Vienna: United Nations publications; 2018. Recuperado de https:/ / www.unodc.org/wdr2018/.

Oksanen, T., Kivimäki, M., Pentti, J., Virtanen, M., Klaukka, T. y Vahtera, J. (2010). Self-report as an indicator of incident disease. Annals of Epidemiology, 20, 547-554. doi:10.1016/j.annepidem.2010.03.017.

Organización Mundial de la Salud. (2016). Prevalence of tobacco smoking. Age standardized prevalence of current tobacco smoking among persons aged 15 years and older (\%), 2015: Male y Female. Recuperado de http:// gamapserver.who.int/gho/interactive_charts/tobacco/ use/atlas.html.

Organización Mundial de la Salud. (2016). The health and social effects of nonmedical cannabis use. Recuperado de https://www.who.int/substance_abuse/publications/ msbcannabis.pdf.

Organización Mundial de la Salud. (2019). Cannabis. Recuperado de https://www.who.int/substance_abuse/ facts/cannabis/en/.

Racz, S. J., Saha, S., Trent, M., Adger, H., Bradshaw, C. P., Goldweber, A. y Cauffman, E. (2016). Polysubstance use among minority adolescent males incarcerated for serious offenses. Child Youth Care Forum, 45, 205-220. doi:10.1007/s10566-015-9334-x.

Rew, L., Taylor-Seehafer, M.y Fitzgerald, M. L. (2001). Sexual abuse, alcohol and other drug use, and suicidal behaviors in homeless adolescents. Issues in Comprehensive Pediatric Nursing, 24, 225-240. doi:10.1080/014608601753260326.

Rial, A., Burkhart, G., Isorna, M., Barreiro, C., Varela, J. y Golpe, S. (2019). Cannabis use among adolescents: Risk pattern, implications and possible explanatory variables. Adicciones, 31, 64-77. doi:10.20882/adicciones.1212.

Roche, D. J., Bujarski, S., Green, R., Hartwell, E. E., Leventhal, A. M. y Ray, L. A. (2019). Alcohol, tobacco, and marijuana consumption is associated with increased odds of same-day substance co-and tri-use. Drug and Alcohol Dependence, 200, 40-49. doi:10.1016/j.drugalcdep.2019.02.035.
Salazar-De La Cuba, A. L., Ardiles-Paredes, D. F., Araujo-Castillo, R. V. y Maguiña, J. L. (2019). High prevalence of self-reported tuberculosis and associated factors in a nation-wide census among prison inmates in Peru. Tropical Medicine E International Health 24, 328338. doi:10.1111/tmi.13199.

Terry-McElrath, Y. M., O’Malley, P. M. y Johnston, L. D. (2013). Simultaneous alcohol and marijuana use among US high school seniors from 1976 to 2011: Trends, reasons, and situations. Drug and Alcohol Dependence, 133, 7179. doi:10.1016/j.drugalcdep.2013.05.031.

Tyler, K. A., Gervais, S. J. y Davidson, M. M. (2013). The relationship between victimization and substance use among homeless and runaway female adolescents. Journal of Interpersonal Violence, 28, 474-493. doi:10.1177/0886260512455517.

Vega-Cauich, J. I., Heredia, B. N. y García, F. M. (2018). Marihuana y drogas ilícitas en jóvenes mexicanos: Factores de riesgo y protección asociados a la edad de inicio del consumo. Revista Iberoamericana de Psicología, 11, 3-10. doi:10.33881/2027-1786.rip.11103.

Velleman, R. D., Templeton, L. J. y Copello, A. G. (2005). The role of the family in preventing and intervening with substance use and misuse: A comprehensive review of family interventions, with a focus on young people. Drug and Alcohol Review, 24, 93-109. doi:10.1080/09595230500167478.

Volkow, N. D., Baler, R. D., Compton, W. M. y Weiss, S. R. (2014). Adverse health effects of marijuana use. The New England Journal of Medicine, 370, 2219-2227. doi:10.1056/ NEJMra1402309.

Wang, S. H., Chen, W. C., Lew-Ting, C. Y., Chen, C. Y. y Chen, W. J. (2010). Running away experience and psychoactive substance use among adolescents in Taiwan: Multi-city street outreach survey. BMC Public Health, 10, 29. doi:10.1186/1471-2458-10-29.

Wechsberg, W. M., Doherty, I. A., Browne, F. A., Kline, T. L., Carry, M. G., Raiford, J. L. y Herbst, J. H. (2015). Gang membership and marijuana use among African American female adolescents in North Carolina. Sub stance Abuse and Rehabilitation, 6, 141-150. doi:10.2147/ SAR.S93304.

Zapata Roblyer, M. I., Betancourth, S. y Grzywacz, J. G. (2015). Risk and protective factors for lifetime marijuana use among Colombian emergent adults attending college. ISSBD Bulletin, 2015, 5-9. 
